\title{
Apogossypolone induces reactive oxygen species accumulation and controls cell cycle progression in Raji Burkkit's lymphoma cells
}

\author{
ZHE-YU HU $^{1 *}$, FEI XU ${ }^{1 *}$, RUI SUN ${ }^{1}$, YAN-FENG CHEN $^{1}$, DONG-SHENG ZHANG ${ }^{1}$, \\ YU-HUA FAN ${ }^{2}$ and JIAN SUN ${ }^{1}$ \\ ${ }^{1}$ Sun Yat-sen University Cancer Center, State Key Laboratory of Oncology in South China, \\ Collaborative Innovation Center for Cancer Medicine, Guangzhou, Guangdong 510060; \\ ${ }^{2}$ The First Affiliated Hospital, Sun Yat-sen University, Guangzhou, Guangdong 510080, P.R. China
}

Received May 3, 2014; Accepted February 5, 2015

DOI: $10.3892 / \mathrm{mmr} .2015 .3404$

\begin{abstract}
Burkitt's lymphoma (BL) is a highly aggressive type of non-Hodgkin's lymphoma, with marked rates of proliferation and metabolism. The expression levels of the translocated cellular Myc (c-Myc) oncogene and Epstein-Barr virus infection have an oncogenic role in facilitating tumor progression and maintaining a malignant phenotype in BL Raji cells. The present study identified that more reactive oxygen species (ROS) were produced in Raji cells compared with other types of malignant B cells. Cells exhibiting higher ROS levels suggested facilitation of the induction of cell death by ROS-induction compounds. In the present study, apogossypolone (ApoG2) was observed to induce marked accumulation in the levels of ROS in the Raji cells, which damaged the cells and suppressed cell proliferation. Within $12 \mathrm{~h}$ following ApoG 2 treatment, the Raji cells were prominently arrested in the G1 phase of the cell cycle. Immunoblotting analysis indicated that the chromodomain-helicase-DNA-binding protein 1, checkpoint kinase 1 and c-Myc proteins were significantly downregulated at 3, 6 and $12 \mathrm{~h}$, respectively, following treatment. Following treatment with ApoG2 for 48 h, ApoG2 induced significant apoptosis in the Raji cells. This findings, together with our previous studies, which demonstrated ApoG2 as a potent inhibitor of anti-apoptotic B cell lymphoma 2 proteins, indi-
\end{abstract}

Correspondence to: Dr Yu-Hua Fan, The First Affiliated Hospital, Sun Yat-sen University, 58 Zhongshan Er Road, Guangzhou, Guangdong 510080, P.R. China

Email: fanyuhua@mail.sysu.edu.cn

Dr Jian Sun, Sun Yat-sen University Cancer Center, State Key Laboratory of Oncology in South China, Collaborative Innovation Center for Cancer Medicine, 651 Dongfeng Road East, Guangzhou, Guangdong 510060, P.R. China

Email: sunjian@sysucc.org.cn

*Contributed equally

Key words: apogossypolone, reactive oxygen species, cell cycle, Burkitt's lymphoma cated that the ROS stimulatory effect of ApoG2 increased the antitumor activity of ApoG2.

\section{Introduction}

Human Burkitt's Lymphoma (BL) originates from the lymphatic system and was first described in children in central Africa in 1956 (1). Immunodeficiency, Epstein-Barr virus (EBV) infection and c-Myc gene translocation have been associated with the development of this malignant disease (2-4). In the human BL Raji cell line, EBV DNA has been reported to integrate into the host genome, leading to identification and isolation of this virus (5). Despite the marked potency of the prevalent regimen of dose-adjusted treatment with etoposide, prednisone, vincristine, cyclophosphamide, doxorubin and rituximab in suppressing Burkitt's lymphoma (6), the majority of patients succumb to mortality within five years.

EBV is a ubiquitous human herpes-virus, which is associated with the development of lymphoid and epithelial tumors (3). The transforming effects of EBV are dependent on its restricted expression of latent proteins in the virally-infected epithelial cells and B-lymohoblastoid cell lines (7,8). LMP1 is the major transforming protein of EBV, which has been observed to act as a classical oncoprotein in rodent fibroblast transforming assays, and is essential for EBV-induced transformation (9). Previously, EBV-induced oxidative stress in B lymphocytes and epithelial cells has been reported to be involved in viral transformation (10). In addition, EBV-positive BL cells have been found to exhibit higher levels of reactive oxygen species (ROS) compared with EBV-negative BL cells, and latent membrane protein 1 (LMP1) is considered to be a major inducer of ROS (11). As a functional homologue of CD40, a member of the tumor necrosis factor (TNF) receptor family, LMP1 can constitutively activate the TNF pathway and, therefore, stimulate a number of downstream signaling pathways, including nuclear factor $(\mathrm{NF})-\kappa \mathrm{B}$, mitogen-activated protein kinase (MAPK) and phosphatidylinositol 3-kinase (PI3K)/Akt (12-15). Ha and Lee (16) demonstrated that the activation of CD40 produces ROS by activating the NADPH oxidase (NOX) regulatory subunit, p40phox, through TNF receptor-associated factor 3 and the PI3K pathway. These findings imply that NOX may be involved in LMP1-induced 
ROS induction in human malignancy, however, the detailed molecular mechanism remains to be elucidated. The NOX family is an important intrinsic source of ROS. By consuming NADPH, NOX catalyzes the generation of superoxide from oxygen, and the activation of NOX has been associated with development of various types of tumor, including human prostate cancer $(17,18)$, melanoma (19) and glioblastoma (20). In the EBV-induced malignant transformation and tumor progression of B-cell lymphoma cells, EBV nuclear antigen (EBNA)-1 has been suggested to activate NOX2/gp91(phox) and facilitate ROS production (21).

ROS accumulation in malignant cells leads to their increased sensitivity to ROS-induction anticancer compounds, including $\beta$-phenylethyl isothiocyanate and cisplatin $(22,23)$. Our previous study demonstrated that the small molecular compound, apogossypolone (ApoG2), not only inhibits the B cell lymphoma (Bcl)-2 anti-apoptotic protein, but also induce ROS generation in nasopharyngeal carcinoma cells $(24,25)$. The present study detected ROS levels, and NAPDH oxidase activity in EBV-positive Raji cells and EBV-negative malignant B cells. In addition, ROS accumulation, and cell cycle progression and regulation were analyzed following treatment with ApoG2.

\section{Materials and methods}

Cell lines, chemicals and reagents. The EBV-positive Raji cells, a well-established human BL cell line with EBV-latent infection; human pre-B leukemic 697 cells, human hematopoietic B NALM 6 cells and acute lymphoblastic leukemia-derived REH cells were maintained in the Sun Yat-sen University Cancer Center laboratory (Guangzhou, China) in RPMI-1640 (Gibco-BRL, Gaithersburg, MD, USA), supplemented with $10 \%$ heat-inactivated fetal bovine serum (Thermo Fihser Scientific, HyClone, Logan, UT, USA). The cells were incubated in a humidified $5 \% \mathrm{CO}_{2}$ atmosphere at $37^{\circ} \mathrm{C}$. Apogossypolone (ApoG2) was provided by Professor Dajun Yang (Ascenta Therapeutics, Inc., Malvern, PA, USA) and was dissolved in dimethyl sulfoxide (DMSO) and freshly diluted in RPMI-1640 prior to used. The final DMSO concentration was $<0.1 \%(\mathrm{v} / \mathrm{v})$. 2',7'-dichlorodihydrofluorescein diacetate (CM-H2DCF-DA) was purchased from Invitrogen Life Technologies, Carlsbad, CA), USA.

Flow cytometry. Raji, REH, 697 and NALM16 cells $\left(5 \times 10^{6}\right)$ were treated by $10 \mu \mathrm{M}$ ApoG2 for $3 \mathrm{~h}$, then the cellular ROS contents were measured by incubating the drug-treated Raji, REH, 697 and NALM16 cells with $1 \mu \mathrm{M}$ CM-H2DCF-DA for $60 \mathrm{~min}$ at $37^{\circ} \mathrm{C}$, followed by flow cytometry, using a FACSCalibur equipped with CellQuest Pro software version 3.3 (BD Biosciences, Franklin Lakes, CA, USA). CM-H2DCF-DA is a fluorescent probe, which is relatively specific for hydrogen peroxide (38). To determine the effect of ApoG2 on cell cycle, Raji cells were treated with $10 \mu \mathrm{M}$ ApoG2 for 8 and $16 \mathrm{~h}$. Then untreated and drug-treated Raji cells $\left(5 \times 10^{6}\right)$ were harvested. Propidium iodide (PI) staining was performed following $75 \%$ alcohol fixation, which was then analyzed using flow cytometry. The cellular DNA content was determined using a flow cytometer (FC 500 MCL; Beckman Coulter, Brea, CA, USA). Apoptotic cells were identified by the sub-G1 phase in the cell cycle distribution.
NOX activity assay. DPI is widely used as an inhibitor of flavoenzymes, particularly NADPH oxidases $(19,20)$. To determine the cellular NOX activity, $10 \mu \mathrm{M}$ DPI (Sigma-Aldrich, St. Louis, MO, USA) was added $4 \mathrm{~h}$ prior to harvesting the cells. The control and DPI-treated Raji and NALM6, 697 and REH cells $\left(5 \times 10^{6}\right)$ were lysed in hypotonic phosphate buffer containing protease inhibitors (Sigma-Aldrich), and were then disrupted by sonication (Q125; Qsonica LLC, Newtown, CT, USA), centrifuged for $10 \mathrm{~min}$ at $200 \mathrm{x} \mathrm{g}$. The supernatant, which contained the cytosol and mitochondrial fraction, was further ultra-centrifuged at $100,000 \mathrm{x}$ g for $30 \mathrm{~min}$ at $4^{\circ} \mathrm{C}$. The resulting pellet, containing the membranous fraction of cytosol and mitochondria, was resuspended in buffer B, containing $50 \mathrm{mM}$ Tris (pH 7.5), $150 \mathrm{mM} \mathrm{NaCl}, 1 \mathrm{mM}$ EDTA, protease inhibitor cocktail (one tablet for $10 \mathrm{ml}$ buffer; Thermo Fisher Scientific, Waltham, MA, USA) for the NOX activity assay. The samples were adjusted to each contain the same concentration of proteins $(1 \mu \mathrm{g} / \mu \mathrm{l})$, and $5 \mu \mathrm{l}$ of each sample was incubated with $94 \mu \mathrm{l}$ phosphate buffer, containing $50 \mathrm{mM} \mathrm{K}_{2} \mathrm{HPO}_{4}$, $1 \mathrm{mM}$ EGTA, $150 \mathrm{mM}$ sucrose, and $1 \mu \mathrm{l}$ 3-NADPH (4 mM) for $15 \mathrm{~min}$, and $2.5 \mu \mathrm{l}$ lucigenin ( $2 \mathrm{mM}$; Anaspec, Inc., Fremont, CA, USA) was added prior to analysis. The lucigenin-derived chemiluminescence of the cell homogenates were assessed over a period of $1 \mathrm{~min}$ in a 20/20 n Tube Luminometer (Turner Biosystem, Sunnyvale, CA).

Assays for cytotoxicity. The cell viability was measured using a 3-(4,5-dimethylthiazol-2-yl)-5-(3-carboxymethox yphenyl)-2-(4-sulfophenyl)-2H-tetrazolium) (MTS) assay (cat. no. CTB169; Promega Corporation, Madison, WI, USA). MTS, in the presence of phenazien methosulfate, produces a formazan product, which has an absorbance maximum of 490-500 $\mathrm{nm}$ in PBS and was quantified using a spectrophotometer (V-530; JASCO, Tokyo, Japan). The Raji cells were plated in 96-well culture clusters (Costar, Cambridge, MA, USA) at a density of 10,000 cells/ml. Serial dilutions were made from stock solution of ApoG2 to desired concentrations $(0.03,0.3$ and $3 \mu \mathrm{M})$. All the experimental concentrations had three replicates. The percentage absorbance relative to the control was plotted as a linear function of drug concentration.

Western blot analysis. Protein analyses by immunoblotting were performed, as previously described (39), using primary antibodies against c-Myc (cat. no. sc-764; Santa Cruz Biotechnology, Inc. Santa Cruz, CA, USA), chromodomain-helicase-DNA-binding protein 1 (CHD1; cat. no. ab3242; Abcam), checkpoint kinase 1 (Chk1; cat. no. 2345; Cell Signaling Technology, Inc. Danvers, MA, USA), cell division cycle 25A (cdc25A; cat. no. sc-97; Santa Cruz Biotechnology, Inc.), DNA damage-binding protein 1 (DDB1; cat. no. 5428; Cell Signaling Technology, Inc.), cyclin-dependent kinase 2 (CDK2) (cat. no. sc-163; Santa Cruz Biotechnology, Inc.), cyclin E (cat. no. sc-481; Santa Cruz, Inc.), cytochrome $c$ (cat. no. 4272; Cell Signaling Technology, Inc.) and B cell lymphoma-associated X protein (Bax; cat. no. 2772; Cell Signaling Technology, Inc.). The total cell lysates were harvested, electrophoresed by $12 \%$ SDS-polyacrylamide gel (Sigma-Aldrich) electrophoresis and then transferred onto polyvinylidene difluoride membranes (Roche, Basel, Switzerland). The membranes were incubated with the antibodies targeting 
c-Myc, CHD1, Chk1, cdc25A, CDK2, cyclin E, cytochrome $c$ and $\operatorname{Bax}\left(1: 1,000\right.$ dilution in blocking buffer) overnight at $4^{\circ} \mathrm{C}$ with gentle agitation. Following incubation with the primary antibodies, the membranes were washed in tris-buffered saline-Tween 20 and incubated with anti-rabbit (\#7071) and anti-mouse (\#7072) horseradish peroxidase-conjugated secondary antibodies (Cell Signaling Technology, Inc.; 1:2000 dilution in blocking buffer) for $1 \mathrm{~h}$ at room temperature, to facilitate detection, and enhanced chemiluminescence reagent (Cell Signaling Technology, Inc.) to develop the blots.

Detection of cell apoptosis. The ApoG2-induced apoptosis in the Raji cells was evaluated using 4,6-diamidine-2-phenylindole (DAPI; Beyotime, Shanghai, China) nuclear staining. The cells were cultured in 6 -well cell culture clusters at $37^{\circ} \mathrm{C}$ and exposed to $0,5,10$ and $20 \mu \mathrm{M}$ ApoG 2 for $48 \mathrm{~h}$. For fluorescent microscopic examination, the cells were fixed with $10 \%$ absolute methanol, permeabilized using $0.5 \%$ Triton-X-100, and stained with DAPI $(2 \mu \mathrm{g} / \mathrm{ml})$ for $30 \mathrm{~min}$ at $37^{\circ} \mathrm{C}$. Subsequently, morphological changes of the nuclei were examined, to assess for apoptotic characteristics by fluorescence microscopy (DFC480; Leica Microsystems, Heidelberg GmbH, Mannheim, Germany).

Statistical analysis. All statistical analyses, to determine the significance of the data, were performed using one way analysis of variance with SPSS 19.0 software (SPSS, Inc., St. Louis, MO, USA). $\mathrm{P}<0.05$ was considered to indicate a statistically significant difference.

\section{Results}

Excessive generation of ROS and NOX activation in EBV-positive Raji lymphoma cells. Increased ROS generation is common in cancer cells with an active glycolytic rate under the influence of oncogenic signals, including EBV infection. It has been demonstrated that EBNA-1 activates NOX2/gp91phox and is responsible for excessive ROS production (21). This ROS accumulation renders cells vulnerable to further oxidative damage by exogenous agents. To investigate the role of EBV on cellular ROS levels and NOX activation in lymphocytes, three EBV-negative lymphocytes, 697, NALM 6 and REH, and EBV-positive Raji cells were used. As shown in Fig. 1A, the flow cytometric analysis indicated that the Raji BL cells exhibited markedly higher basal ROS levels compared with the REH, 697 and NALM6 cells (7-, 9.7- and 35.9-fold, respectively; Fig. 1A). Consistent with the this, the NOX enzyme activity in the Raji cells was also 2.0-, 2.5- and 5.5-fold higher compared with the NALM6, 697 and REH cells, respectively (Fig. 1B). As EBNA1 initiates latent viral replication in B cells and regulates the transcription of other EBV-encoded latent genes, this viral protein is required for EBV latent infection and transformation in all EBV associated tumors (26). A previous study demonstrated that EBNA-1 is able to activate NOX2/gp91(phox) in EBV-associated B-cell lymphoma cells (21). Based on these findings, the present study hypothesized that EBV was able to induce excessive ROS generation in EBV-related BL cells by activating NOX enzyme activity. Excessive ROS levels in Raji BL cells facilitate cell proliferation and tumorigenesis, making cancer cells exhibiting high levels of ROS more sensi- tive to ROS-stimulatory anticancer compounds (10). As shown in Fig 1C, the small-molecular compound, ApoG2, induced the generation of ROS $>10$-fold within $3 \mathrm{~h}$ following treatment in the Raji BL cells. This marked increase in cellular ROS accumulation was hypothesized to result in severe damage to cells.

NOX inhibitor attenuates the ApoG2-induced accumulation of ROS. To further assess the effect of cellular ROS levels on cell proliferation in Raji cells, the cells were pretreated with DPI, a specific inhibitor of the NOX enzyme, prior to treatment with ApoG2. The flow cytometric analysis revealed that $10 \mu \mathrm{M}$ ApoG2 induced a significant increase in ROS (10.7-fold) in the Raji cells, however, this increase was significantly lower following pretreatment with DPI compared with the cells without DPI pretreatment. ApoG2 induced lower levels of ROS in cells pretreated with $5 \mu \mathrm{M}$ DPI at 16 and $24 \mathrm{hr}$ (6.2- and 4.0-fold, respectively; Fig. 2A). DPI not only attenuated the stimulatory effect of ApoG2 on ROS, but also inhibited the antiproliferative activity of ApoG2 in the Raji cells. As shown in Fig. 2B, treatment with either DPI or ApoG2 alone exhibited dose-dependent growth inhibition in the Raji cells following $72 \mathrm{~h}$ incubation, however, when combined with DPI, ApoG2 inhibited growth less compared with that observed without DPI. These results suggested that, by inhibiting NOX, DPI attenuated the ROS-stimulatory effect of ApoG2 and weakened the antiproliferative effect of ApoG2 in the Raji BL cells.

ApoG2 treatment arrests Raji cells in the G1 phase of the cell cycle. Since ApoG2 treatment was observed to significantly suppress cell proliferation (Fig. 2B), the present study investigated the effect of ApoG2-induced ROS accumulation on cell proliferation in the Raji BL cells. To examine the cell cycle proliferation, the cells were treated with $10 \mu \mathrm{M}$ ApoG2 for 8 and $16 \mathrm{~h}$. PI staining and flow cytometric analysis demonstrated that ApoG2 was also unable to induce apoptotic cell death within $16 \mathrm{~h}$ incubation, evidenced by low sub-G1 signaling following PI-staining (Fig. 3A). Compared with $48 \%$ G1-cells in the untreated control, almost 60 and $70 \%$ of the Raji cells were arrested in the G1 phase of the cell cycle following treatment with $10 \mu \mathrm{M}$ ApoG2 at 8 and $16 \mathrm{hr}$, respectively (Fig. 3A and B). These data, indicated that ApoG2 stimulated ROS accumulation in the Raji BL cells within $3 \mathrm{~h}$. and this modification caused significant arrest at the G1 phase of the cell cycle within 16 h. Although ApoG2 did not lead to cell death in the short period of time following treatment, ApoG2 effectively suppressed cell proliferation, which was hypothesized to cause significant cell death.

Cdh1, Chk1 and c-Myc cell cycle regulators are involved in ApoG2-induced cell cycle arrest. To further investigate the molecular mechanism underlying the ApoG2-induced cell cycle arrest, a panel of cell cycle regulators were analyzed using immunoblotting. As shown in Fig. 4A, the immunoblots demonstrated that ApoG2 induced a significant downregulation of c-Myc in the Raji cells, which was consistent with our previous findings in NPC cells and U937 cells (27,28). C-Myc appears to be sensitive to ROS stress, is non-specific (29), and is not only affected by a decrease in ROS, but also by ROS elevation, including ApoG2 treatment (25). In addition to c-Myc, ApoG2 also suppressed the expression levels of Cdh1 and Chk1 
A
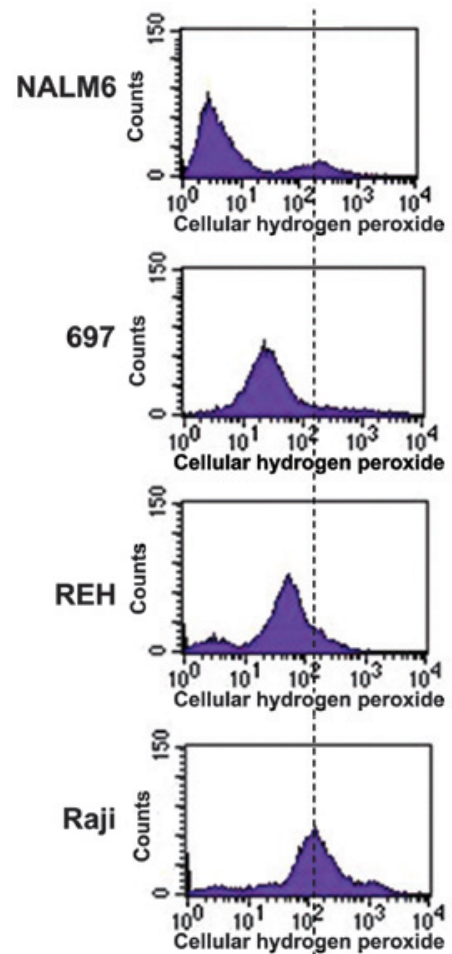

B

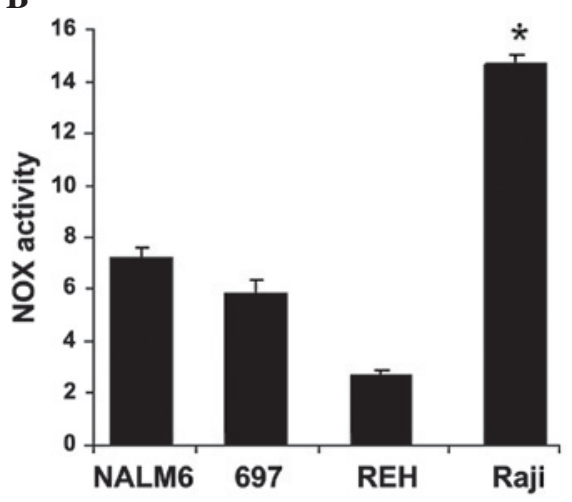

C

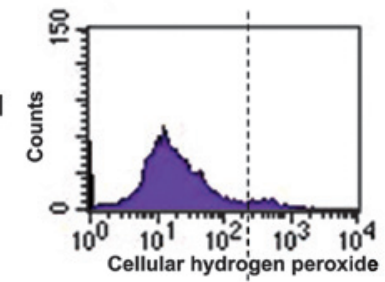

ApoG2

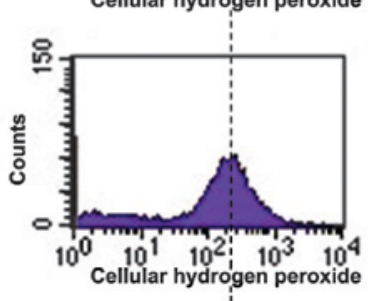

Figure 1. Activation of NOX in EBV-positive Raji lymphoma cells. (A) Comparison of cellular ROS content in the NALM6, 697, REH and Raji cells. Each histogram is representative of three experiments ( $\mathrm{P}<0.001$, Raji cells, compared with. REH cells). (B) Comparison of NOX activity in the NALM6, 697, REH and Raji cells. Data are expressed as the mean \pm standard deviation of three experiments ("P $<0.01$ Raji cells, compared with REH cells). (C) ApoG2 induced cellular ROS accumulation in the Raji cells in $3 \mathrm{~h}$. Each histogram is representative of three experiments $(\mathrm{P}<0.001$ compared with the untreated Raji cells). ApoG2, apogossypolone; NOX, NADPH oxidase; CM-H2DCF-DA, 2',7'-dichlorodihydrofluorescein diacetate.

A

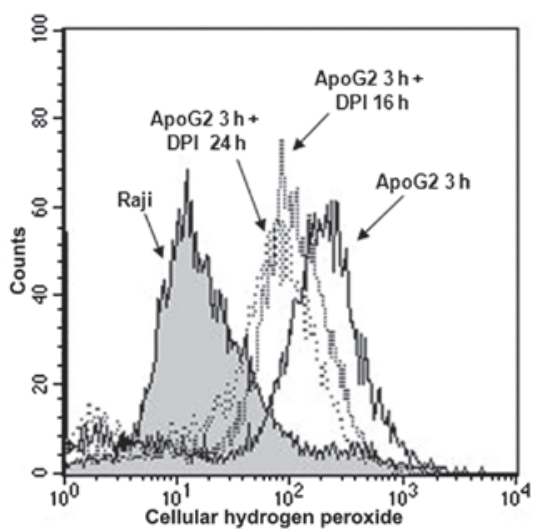

B

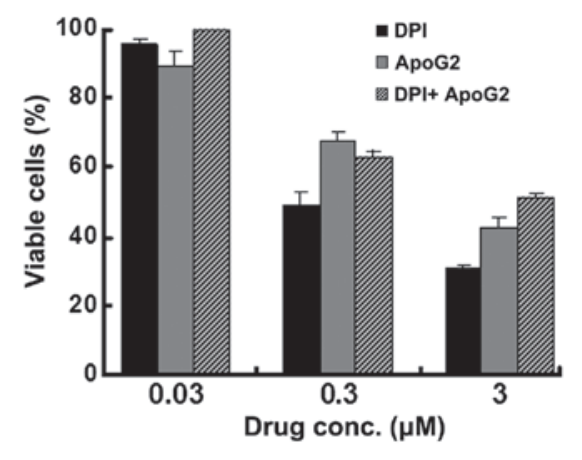

Figure 2. Inhibition of NOX activity and ROS by DPI attenuates the antiproloferative effect of ApoG2 in Raji cells. (A) Effect of DPI on ApoG2-induced ROS accumulation (pretreatment with DPI for 16 and $24 \mathrm{~h}$ ). Each histogram is representative of three experiments. (B) Effects of the combination of ApoG2 and DPI on the growth of the Raji cells in vitro after $72 \mathrm{~h}$. The data are expressed as the mean \pm standard deviation of three experiments; bars. ApoG2, apogossypolone; DPI, diphenyleneiodonium; CM-H2DCF-DA, 2',7'-dichlorodihydrofluorescein diacetate.

as early as 3 and $6 \mathrm{~h}$ following treatment (Fig. 4B). However, ApoG2 did not cause significant changes in the expression levels of cdc25, DDB1, cyclin E and CDK2 in the Raji BL cells. Cdh1 has been reported to induce G1/S arrest. These results suggested that the effects of ApoG2 on cell cycle proliferation in Raji cells was likely mediated by a pattern of modification of cell cycle regulators, including Cdh1, Chk1 and c-Myc.
ApoG2 treatment induced apoptosis in Raji BL cells. As the results of the PI staining and flow cytometry indicated that ApoG2 did not induce cell death, but caused significant cell cycle arrest within $16 \mathrm{~h}$ of treatment, the present study examined weather the early damage resulted in cell death at a later stage. To confirm this hypothesis, 5-20 $\mu \mathrm{M} \mathrm{ApoG} 2$ was used to treat cells for $48 \mathrm{~h}$. As shown in Fig. 5A, PI staining 
A

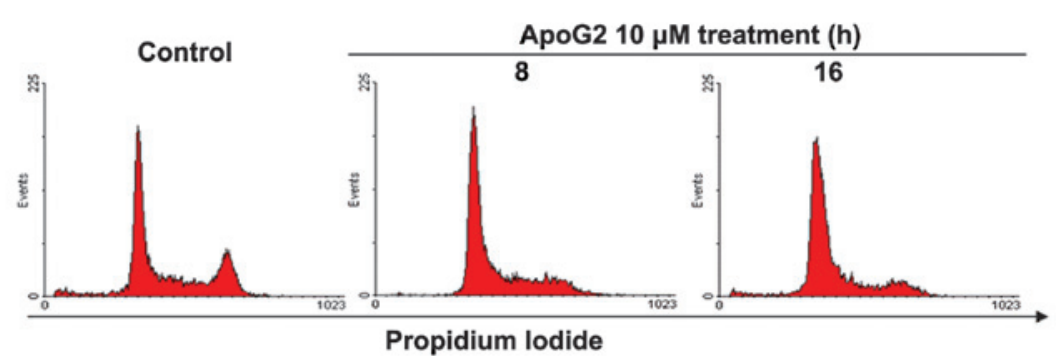

B

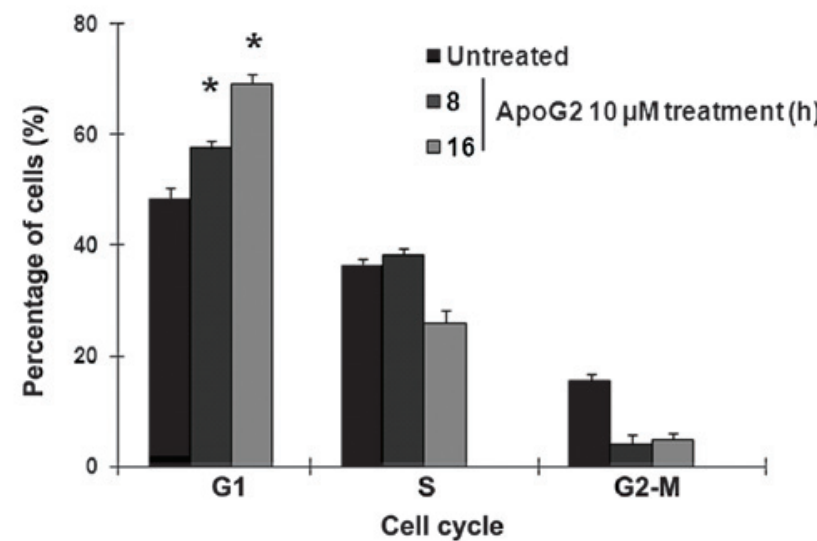

Figure 3. ApoG2 treatment arrests Raji cells in the G1 phase of the cell cycle. (A) Cell cycle analyses of untreated Raji cells and Raji cells treated with $10 \mu \mathrm{M}$ ApoG2 for 8 and $16 \mathrm{~h}$. Each histogram is representative of three experiments. (B) Analysis of cell cycle distributions following ApoG2 treatment. The data are expressed as the mean \pm standard deviation of three independent experiments. " $\mathrm{P}<0.05$, compared with the untreated cells. ApoG2, apogossypolone.

A

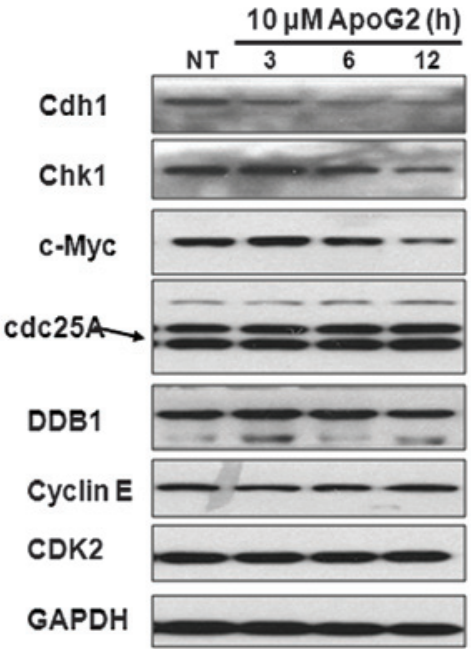

B

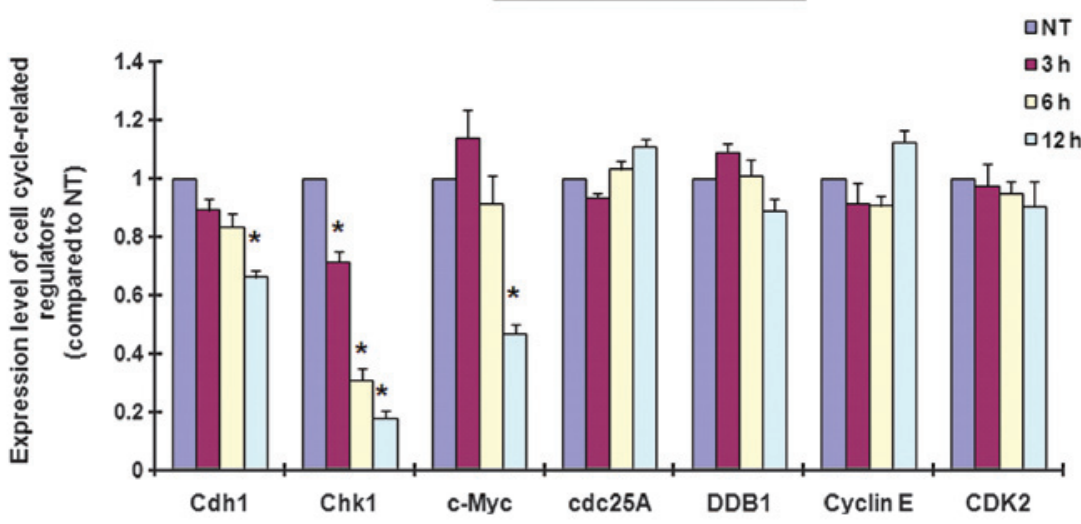

Figure 4. Effects of ApoG2 on cell cycle regulators. (A) Immunoblot demonstrating the modulation of cell cycle regulators in ApoG2-treated Raji cells. (B) Analysis of the protein expression levels of C-Myc, Chk1 and CDH1 following ApoG2 treatment. Data are expressed as the mean \pm standard deviation of three experiments. "P $<0.05$, expression levels of cell cycle regulators compared with NT. ApoG2, apogossypolone; Chd1, chromodomain-helicase-DNA-binding protein 1; Chk1, checkpoint kinase 1; cdc25A, cell division cycle 25A; DDB1, DNA damage-binding protein 1; CDK2, cyclin-dependent kinase 2. 
A

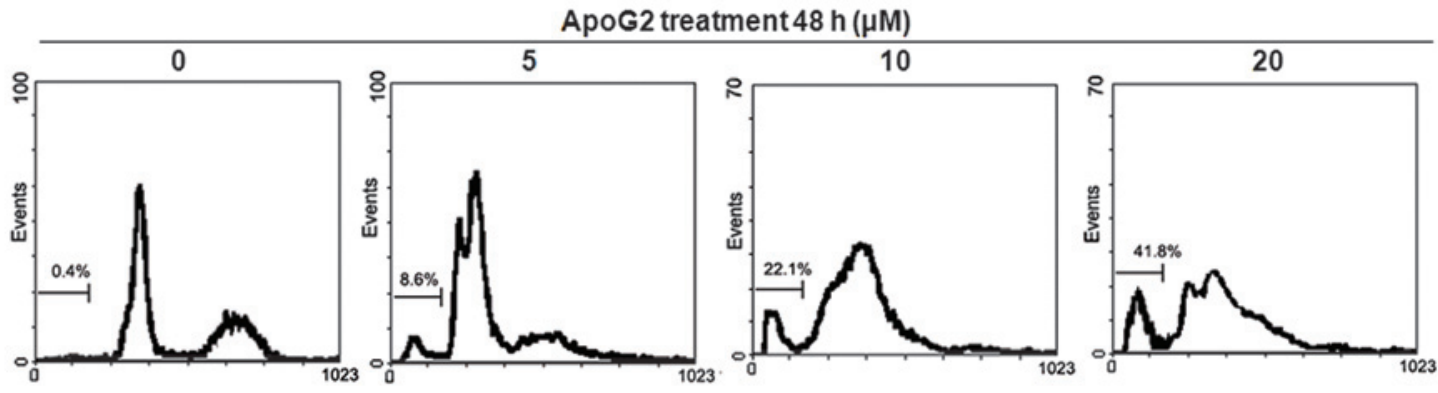

B

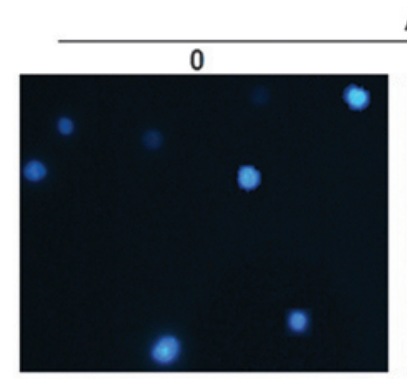

ApoG2 treatment $48 \mathrm{~h}(\mu \mathrm{M})$

C

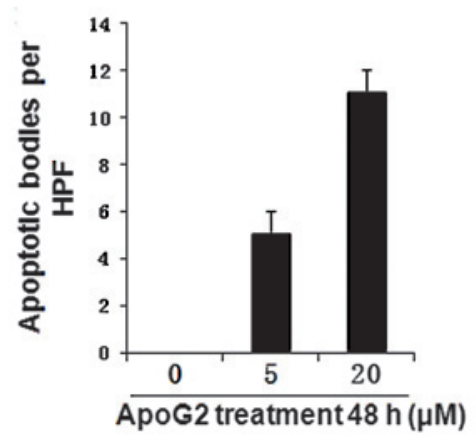

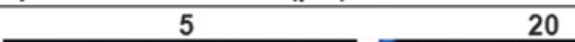
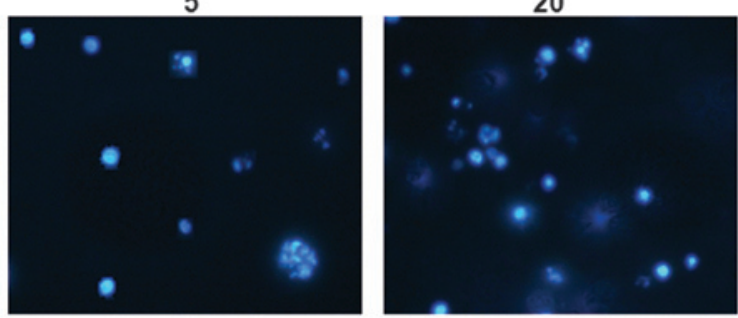

D

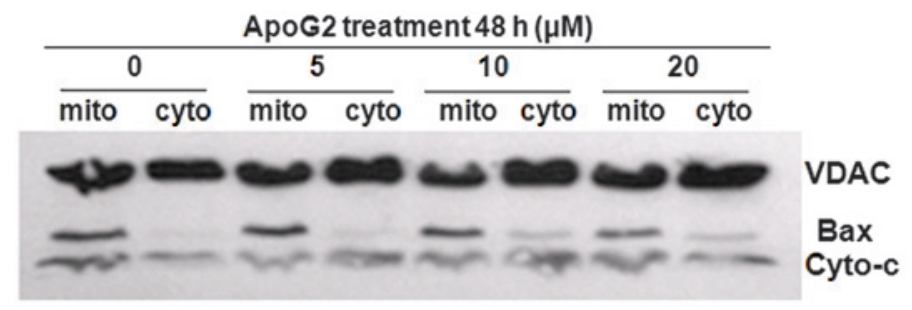

Bax
Cyto-c

Figure 5. ApoG2-induced ROS accumulation triggers apoptosis in Raji cells. (A) The effect of ApoG2 on the sub-G1 population in Raji cells. Each histogram is representative of three experiments. (B) The effect of ApoG2 on nucleosome formation in the Raji cells. Each histogram is representative of three experiments. (C) Number of DAPI-stained nuclei characteristic of apoptosis per $40 \mathrm{HPF}$. Numbers indicate the mean \pm standard deviation of three HPFs per sample and are representative of three independent experiments. (D) The effect of 0-20 M ApoG2 for $48 \mathrm{~h}$ on the mitochondrial and cytosol levels of cytochrome $c$ in the Raji cells. $\beta$-actin served as a loading control. ApoG2, apogossypolone; HPF, high-power field; mito, mitochondrial; Cyto, cytosol; Cyto-c, cytochrome- $C$; Bax, B cell lymphoma 2-associated X protein; DAPI, 4, 6-diamidine-2-phenylindole.

and flow cytometric analysis data indicated that, compared with the untreated control cells, 10 and $20 \mu \mathrm{M}$ ApoG2 induced a significant sub-G1 peak (22 and $41 \%$, respectively) in the Raji BL cells. DAPI staining further demonstrated that 5 and $20 \mu \mathrm{M}$ ApoG 2 treatment for $48 \mathrm{~h}$ caused significant elevation in the number of apoptotic cells with condensed chromatin (Fig. 5B andC). To confirm the ApoG2-induced cell apoptosis in the Raji BL cells, immunobloting was performed to examine the release of cytochrome $c$ and Bax from the mitochondria into cytosol. As shown in Fig. 5D, the immunobloting data demonstrated that cytochrome $c$ was significantly released into the cytosol by $5 \mu \mathrm{M}$ ApoG 2 treatment for $48 \mathrm{~h}$. The levels of Bax in the cytosol also increased in a dose-dependent manner $48 \mathrm{~h}$ after ApoG2 treatment. Taken together, these findings suggested that ApoG2 was able to induce significant apoptotic cell death a relatively long period of time following treatment.

\section{Discussion}

Burkitt's lymphoma (BL) is an aggressive malignant disease with high morbidity and low survival rates. The incidence of this disease has increased significantly worldwide as its development is associated with viral infection and immunodeficiency (30). EBV is a type of human herpes virus, and is associated with the pathogenesis of BL. As with several other oncoviruses, including hepatitis B virus and human T-lymphotropic virus 1, which induce oxidative stress and metabolic disorder in host cells $(31,32)$, EBV has also been found to upregulate cellular ROS levels through the EBNA1-NOX signaling pathway (21). Consistent with these reports, the present study found that, compared with the NALM6, 697 and REH EBV-negative lymphocytes, the EBV-positive Raji cells exhibited significantly higher levels of NOX enzyme activity and cellular ROS. Based on these findings, it was suggested that NOX enzyme activation and elevated ROS levels may be potent targets for anticancer treatment in Raji BL cells or cancer cells with similar characters.

It is understood that cancer cells with higher ROS levels are more sensitive to ROS-stimulatory compounds, including cisplatin and paclitaxel (22-23). In the present study, ApoG2 demonstrated potent effects on ROS stimulation as early as $3 \mathrm{~h}$ after treatment. This significant increase in ROS content is likely to damage cell components, including mitochondria and 
the nuclear and cell membranes. The present study found that, as early as $8 \mathrm{~h}$ after ApoG 2 treatment, almost $60 \%$ of the Raji cells accumulated in the G1 phase of the cell cycle. At $16 \mathrm{~h}$ after treatment, this percentage increased to $70 \%$, although few cells had undergone apoptotic cell death, evidenced by low sub-G1 signal in cells with PI staining. However, a longer treatment duration of $48 \mathrm{~h}$ resulted in a marked sub-G1 peak and apoptotic cell death. These data suggested that the ApoG2-induced ROS accumulation led to cell cycle arrest in the G1 phase at the early stage and caused apoptotic cell death at the late stage.

Cell cycle progression is regulated by a panel of molecules, including CDKs, cyclins, their regulators (33). $\mathrm{Cdh} 1$ is an adapter protein of anaphase-promoting complex (APC) and controls the G0 and G1 phases of the cell cycle. It stabilizes the G1 phase by recognizing mitotic proteins, including cyclins, and recruits them to the APC for ubiquination (34). In neurons, actively upregulated APC/Cdh1 can constantly degrade glycolysis enzyme 6-phosphofructo-2-kinase/fructose-2, 6-bisphosphatase-3 to support aerobic respiration and maintain antioxidant status (35). A previous study also reported that antioxidant treatment arrests cells in the late-G1 phase by upregulating APC/Cdh1 activity (36). Unlike the activation of APC/Cdh1 by antioxidants, the present study demonstrated that the ApoG2-induced ROS accumulation in the Raji BL cells suppressed the expression of Cdh1 and arrested cells in the G1 phase. Chk1 was also significantly suppressed by ApoG2 treatment. Chk1 is a kinase, which phosphorylates cdc25 at Ser 216. Cdc25 is phosphorylated during interphase, but not in mitosis. Phosphorylated cdc25 binds to members of the 14-3-3 family of proteins, which prevents the activation of cdc 2 and prevents cells entering the $\mathrm{M}$ phase of the cell cycle (33-34). The exposure of cells to excessive ROS has been found to deplete cyclin D1 and activate Chk1 (37). In the present study, the protein expression of Chk1 decreased as early as $6 \mathrm{~h}$ after ApoG2 treatment.

The c-Myc cell cycle regulator was also affected by ApoG2 treatment in the present study. Our previous study demonstrated that ApoG2 suppressed the levels of c-Myc in NPC cells and U937 lymphoma cells $(27,28)$. In the present study c-Myc was downregulated by ApoG2 treatment in the Raji BL cells. In addition, the NOX inhibitor, DPI, also suppressed the protein expression of c-Myc within $12 \mathrm{~h}$ of treatment (data not shown), which suggested that the protein suppression of c-Myc was not specific to ApoG2, but was affected by ROS accumulation and depletion.

In conclusion, the results of the present study demonstrated that the EBV-positive Raji BL cells exhibited increased cellular ROS content compared with the EBV-negative lymphocytes, and this ROS elevation facilitated the sensitivity of the Raji cells to the ROS-stimulatory compound, ApoG2. ApoG2 induced a significant increase in the levels of cellular ROS in the Raji cells within $3 \mathrm{~h}$ of treatment. Within $8 \mathrm{~h}$ of treatment, ApoG2 arrested almost $60 \%$ of the Raji cells in the G1 phase of the cell cycle, and the Cdh1 and Chk1 regulators may have been involved. Within 48 h of treatment, ApoG2 induced significant apoptotic cell death in the Raji BL cells. These findings indicate ApoG2 as a promising compound for anti-BL treatment.

\section{References}

1. Davies JN, Elmes S, Hutt MS, Mtimavalye LA, Owor R and Shaper L: Cancer in an African community, 1897 - 1956. An analysis of the records of Mengo hospital, Kampala, Uganda. 2. Br Med J 1: 336-341, 1964.

2. Molyneux EM, Rochford R, Griffin B, et al: Burkitt's lymphoma. Lancet 379: 1234-1244, 2012.

3. Bornkamm GW: Epstein-Barr virus and the pathogenesis of Burkitt's lymphoma: more questions than answers. Int J Cancer 124: 1745-1755, 2009.

4. zur Stadt U, Hoser G, Reiter A, Welte K and Sykora KW: Application of long PCR to detect $\mathrm{t}(8 ; 14)(\mathrm{q} 24 ; \mathrm{q} 32)$ translocations in childhood Burkitt's lymphoma and B-ALL. Ann Oncol 8 (Suppl 1): 31-35, 1997.

5. Ansari MA, Singh VV, Dutta S, et al: Constitutive interferon-inducible protein 16-inflammasome activation during Epstein-Barr virus latency I, II, and III in B and epithelial cells. J Virol 87: 8606-8623, 2013.

6. Dunleavy K, Pittaluga S, Shovlin M, et al: Low-intensity therapy in adults with Burkitt's lymphoma. N Engl J Med 369: 1915-1925, 2013.

7. Young LS and Murray PG: Epstein-Barr virus and oncogenesis: from latent genes to tumours. Oncogene 22: 5108-5121, 2003.

8. Chou YC, Lin SJ, Lu J, et al: Requirement for LMP1-induced RON receptor tyrosine kinase in Epstein-Barr virus-mediated B-cell proliferation. Blood 118: 1340-1349, 2011.

9. Dawson CW, Port RJ and Young LS: The role of the EBV-encoded latent membrane proteins LMP1 and LMP2 in the pathogenesis of nasopharyngeal carcinoma (NPC). Semin Cancer Biol 22: 144-153, 2012.

10. Lassoued S, Ben Ameur R, Ayadi W, Gargouri B, Ben Mansour R and Attia H: Epstein-Barr virus induces an oxidative stress during the early stages of infection in B lymphocytes, epithelial, and lymphoblastoid cell lines. Mol Cell Biochem 313: 179-186, 2008.

11. Cerimele F, Battle T, Lynch R, et al: Reactive oxygen signaling and MAPK activation distinguish Epstein-Barr Virus (EBV)-positive versus EBV-negative Burkitt's lymphoma. Proc Natl Acad Sci USA 102: 175-179, 2005.

12. Kraus ZJ, Nakano H and Bishop GA: TRAF5 is a critical mediator of in vitro signals and in vivo functions of LMP1, the viral oncogenic mimic of CD40. Proc Natl Acad Sci USA 106: 17140-17145, 2009.

13. Gewurz BE, Mar JC, Padi M, et al: Canonical NF-kappaB activation is essential for Epstein-Barr virus latent membrane protein 1 TES2/CTAR2 gene regulation. J Virol 85: 6764-6773, 2011.

14. Shkoda A, Town JA, Griese J, et al: The germinal center kinase TNIK is required for canonical $\mathrm{NF}-\kappa \mathrm{B}$ and JNK signaling in B-cells by the EBV oncoprotein LMP1 and the CD40 receptor. PLoS Biol 10: e1001376, 2012.

15. Shair KH, Bendt KM, Edwards RH, Bedford EC, Nielsen JN and Raab-Traub N: EBV latent membrane protein 1 activates Akt, NFkappaB, and Stat3 in B cell lymphomas. PLoS Pathog 3: e166, 2007.

16. Ha YJ and Lee JR: Role of TNF receptor-associated factor 3 in the CD40 signaling by production of reactive oxygen species through association with $\mathrm{p} 40$ phox, a cytosolic subunit of nicotinamide adenine dinucleotide phosphate oxidase. J Immunol 172: 231-239, 2004.

17. Lim SD, Sun C, Lambeth JD, et al: Increased Nox1 and hydrogen peroxide in prostate cancer. Prostate 62: 200-207, 2005.

18. Huang WC, Li X, Liu J, Lin J and Chung LW: Activation of androgen receptor, lipogenesis, and oxidative stress converged by SREBP-1 is responsible for regulating growth and progression of prostate cancer cells. Mol Cancer Res 10: 133-142, 2012.

19. Yamaura M, Mitsushita J, Furuta S, et al: NADPH oxidase 4 contributes to transformation phenotype of melanoma cells by regulating $\mathrm{G} 2-\mathrm{M}$ cell cycle progression. Cancer Res 69: 2647-2654, 2009

20. Hsieh CH, Shyu WC, Chiang CY, Kuo JW, Shen WC and Liu RS: NADPH oxidase subunit 4-mediated reactive oxygen species contribute to cycling hypoxia-promoted tumor progression in glioblastoma multiforme. PLoS One 6: e23945, 2011.

21. Gruhne B, Sompallae R, Marescotti D, Kamranvar SA, Gastaldello S and Masucci MG: The Epstein-Barr virus nuclear antigen-1 promotes genomic instability via induction of reactive oxygen species. Proc Natl Acad Sci USA 106: 2313-2318, 2009. 
22. Trachootham D, Zhou Y, Zhang H, et al: Selective killing of oncogenically transformed cells through a ROS-mediated mechanism by beta-phenylethyl isothiocyanate. Cancer Cell 10: 241-252, 2006

23. Marullo R, Werner E, Degtyareva N, et al: Cisplatin induces a mitochondrial-ROS response that contributes to cytotoxicity depending on mitochondrial redox status and bioenergetic functions. PLoS One 8: e81162,2013.

24. Hu ZY, Zhu XF, Zhong ZD, et al: ApoG2, a novel inhibitor of antiapoptotic Bcl-2 family proteins, induces apoptosis and suppresses tumor growth in nasopharyngeal carcinoma xenografts. Int J Cancer 123: 2418-2429, 2008.

25. Hu ZY, Wang J, Cheng G, et al: Apogossypolone targets mitochondria and light enhances its anticancer activity by stimulating generation of singlet oxygen and reactive oxygen species. Chin J Cancer 30: 41-53, 2011.

26. Canaan A, Haviv I, Urban AE, et al: EBNA1 regulates cellular gene expression by binding cellular promoters. Proc Natl Acad Sci USA 106: 22421-22426, 2009.

27. Hu ZY, Sun J, Zhu XF, Yang D and Zeng YX: ApoG2 induces cell cycle arrest of nasopharyngeal carcinoma cells by suppressing the c-Myc signaling pathway. J Transl Med 7: 74, 2009.

28. Sun J, Li ZM, Hu ZY, Zeng ZL, Yang DJ and Jiang WQ Apogossypolone inhibits cell growth by inducing cell cycle arrest in U937 cells. Oncol Rep 22: 193-198, 2009.

29. Maciag AE, Holland RJ, Robert Cheng YS, et al: Nitric oxide-releasing prodrug triggers cancer cell death through deregulation of cellular redox balance. Redox Biol 1: 115-124, 2013.

30. Mbulaiteye SM, Anderson WF, Ferlay J, et al: Pediatric, elderly, and emerging adult-onset peaks in Burkitt's lymphoma incidence diagnosed in four continents, excluding Africa. Am J Hematol 87: 573-578, 2012.
31. Gu JM, Lim SO, Oh SJ, Yoon SM, Seong JK and Jung G: HBx modulates iron regulatory protein 1-mediated iron metabolism via reactive oxygen species. Virus Res 133: 167-177, 2008.

32. Silic-Benussi M, Cavallari I, Vajente N, et al: Redox regulation of T-cell turnover by the p13 protein of human T-cell leukemia virus type 1: distinct effects in primary versus transformed cells. Blood 116: 54-62, 2010.

33. Ishidate T, Elewa A, Kim S, Mello CC and Shirayama M: Divide and differentiate: CDK/Cyclins and the art of development. Cell Cycle 13: 1384-1391, 2014.

34. Li M, Shin YH, Hou L, et al: The adaptor protein of the anaphase promoting complex Cdh1 is essential in maintaining replicative lifespan and in learning and memory. Nat Cell Biol 10: 1083-1089, 2008

35. Herrero-Mendez A, Almeida A, Fernández E, Maestre C, Moncada S and Bolaños JP: The bioenergetic and antioxidant status of neurons is controlled by continuous degradation of a key glycolytic enzyme by APC/C-Cdh1. Nat Cell Biol 11: 747-752, 2009.

36. Havens CG, Ho A, Yoshioka N and Dowdy SF: Regulation of late G1/S phase transition and APC Cdh1 by reactive oxygen species. Mol Cell Biol 26: 4701-4711, 2006.

37. Pyo CW, Choi JH, Oh SM and Choi SY: Oxidative stress-induced cyclin D1 depletion and its role in cell cycle processing. Biochim Biophys Acta 1830: 5316-5325, 2013.

38. Pelicano H, Feng L, Zhou Y, et al: Inhibition of mitochondrial respiration: a novel strategy to enhance drug-induced apoptosis in human leukemia cells by a reactive oxygen species-mediated mechanism. J Biol Chem 278: 37832-37839, 2003.

39. Guo C, Pan ZG, Li DJ, et al: The expression of p63 is associated with the differential stage in nasopharyngeal carcinoma and EBV infection. J Transl Med 4: 23, 2006. 